\title{
Ciclo de mejora docente en el Taller de Arquitectura. El modelo urbano como clave para la estrategia de proyecto.
}

\section{Cycle of teaching improvement in the Architecture Workshop. The urban model as a key to the project strategy.}

FRANCISCO JAVIER OStos PRIETO

ORCID: https://orcid.org/0000-0002-9551-3946

Universidad de Sevilla

Departamento de Urbanística y

Ordenación del Territorio

fostos1@us.es

DOI: http://dx.doi.org/10.12795/9788447231003.053

Pp.: 1110-1132 


\section{Descripción del contexto}

Como inicio, es necesario destacar que esta asignatura ha estado marcada por el contexto derivado de la pandemia sufrida en este año de 2020, en el que la docencia universitaria se ha modificado en su totalidad. La Escuela de Arquitectura tomó la decisión de que los grupos de tercero realizaran una docencia semipresencial, es decir, a cada clase asiste siempre el 50\% de los alumnos matriculados, estando el otro 50\% de manera síncrona virtual. Tras haber realizado un modelo $100 \%$ presencial, posteriormente en confinamiento 100\% virtual (Universidad de Sevilla, 2020b), ahora nos volvemos a enfrentar a un nuevo escenario docente en el que muchos aspectos no se llegan a controlar quedando en muchos casos a merced de la situación sanitaria en la que se ha desarrollado el ciclo de mejora en esta asignatura. Además, hay que sumar la imposibilidad de compartir material, mantener distancias con los estudiantes y llevar siempre mascarilla, lo que casi ha derivado en un $90 \%$ el empleo de la plataforma virtual para el desarrollo de clases y exposición de trabajos.

Inserta en el Grado en Fundamentos de la Arquitectura, se encuentra una de las asignaturas troncales, que acompañan al estudiante durante 4 años, denominada Taller de Arquitectura (6 ECTS). En concreto desempeño la docencia en Taller 3, enfocado con el enunciado "equipamiento" (Universidad de Sevilla, 2020a), teniendo lugar en el primer cuatrimestre del tercer curso del grado como profesor en el área de urbanismo.

A diferencia del resto de asignaturas en arquitectura en las que prima una gran carga de contenidos teóricos, el taller se estructura con un enunciado totalmente práctico. En este sentido, los estudiantes deben hacer frente a un proyecto arquitectónico empleando los conocimientos que son adquiridos a través de las demás asignaturas.

Ciclos de Mejora en el Aula (2020). Experiencias de Innovación Docente de la US Esta obra se distribuye con la licencia Creative Commons 
El Taller de Arquitectura se configura como una asignatura multidisciplinar en sí misma, en la que diferentes profesores pertenecientes a 6 departamentos de áreas de conocimiento distintas (Universidad de Sevilla, 2020a) trabajan en equipo para que el estudiante pueda aplicar los conceptos necesarios en el proyecto. Aunque son múltiples docentes los que imparten docencia en la asignatura, la coordinación recae sobre el área de Proyectos Arquitectónicos. Así, se realiza una propuesta de cronograma en el calendario (Tabla 1), según las horas asignadas para cada profesor, de modo que nos vamos cruzando y asistiendo a clase de manera individual o conjunta con otros compañeros docentes.

Tabla 1. Cronograma del Taller de Arquitectura 3. Las siglas en las sesiones representan a los profesores por áreas: Proyectos (P), Expresión Gráfica (EG), Urbanismo (U),

Estructuras (E), Instalaciones (I) y Construcción (C).

\begin{tabular}{cccc} 
& & Sesión 1 & Sesión 2 \\
& & $9: 00-11: 00$ & $11: 30-13: 30$ \\
Fase 1 - Análisis & Semana 1 & $\mathrm{P} / \mathrm{EG} / \mathbf{U} / \mathrm{E} / \mathrm{I} / \mathrm{C}$ & $\mathrm{P}$ \\
& Semana 2 & $\mathrm{P}$ & $\mathrm{P}$ \\
& Semana 3 & $\mathbf{U} / \mathrm{P}$ & $\mathrm{P} / \mathrm{C}$ \\
\cline { 2 - 4 } Fase 2 - Ideación & Semana 4 & $\mathrm{P} / \mathrm{I}$ & $\mathrm{P} / \mathbf{U}$ \\
& Semana 5 & $\mathbf{U} / \mathrm{EG}$ & $\mathrm{P} / \mathrm{EG}$ \\
& Semana 6 & $\mathrm{P} / \mathrm{EG}$ & $\mathrm{P} / \mathbf{U}$ \\
& Semana 7 & $\mathrm{P} / \mathrm{EG} / \mathbf{U} / \mathrm{E} / \mathrm{I} / \mathrm{C}$ & $\mathrm{P} / \mathrm{EG} / \mathbf{U} / \mathrm{E} / \mathrm{I} / \mathrm{C}$ \\
\cline { 2 - 4 } Fase 3 - Desarrollo & $\mathrm{P}$ & $\mathrm{C}$ \\
& Semana 8 & $\mathrm{P}$ & $\mathrm{I} / \mathrm{C}$ \\
& Semana 9 & $\mathrm{C} / \mathrm{EG}$ \\
& Semana 11 & $\mathrm{P} / \mathrm{EG} / \mathbf{U} / \mathrm{E} / \mathrm{I} / \mathrm{C}$ & $\mathrm{P} / \mathrm{EG} / \mathbf{U} / \mathrm{E} / \mathrm{I} / \mathrm{C}$ \\
& Seman 12 & $\mathrm{P} / \mathrm{E}$ & $\mathrm{C} / \mathrm{E}$ \\
& Semana 13 & $\mathrm{P} / \mathrm{E}$ & $\mathrm{P} / \mathrm{C} / \mathrm{E}$ \\
& Semana 14 & $\mathrm{P} / \mathrm{EG} / \mathbf{U} / \mathrm{E} / \mathrm{I} / \mathrm{C}$ & $\mathrm{P} / \mathrm{EG} / \mathbf{U} / \mathrm{E} / \mathrm{I} / \mathrm{C}$
\end{tabular}

Ciclos de Mejora en el Aula (2020). Experiencias de Innovación Docente de la US Esta obra se distribuye con la licencia Creative Commons 
Al ocupar el área de urbanística, esta queda inserta con mayor carga al inicio de la asignatura, según el programa docente realizado por el coordinador de la asignatura. En este, el modelo metodológico se define 3 fases diferenciadas: 1a fase - Análisis, 2a fase - Ideación y 3a fase - Desarrollo (Figura 1). La idea principal es que a partir de un problema planteado, la realización de un proyecto de equipamiento hospitalario con programa mixto, se pretende que los estudiantes analicen en primer lugar el contexto donde se ubica, estudien ejemplos relacionados y propongan ideas, para finalmente concretar sus propias propuestas. A su vez, el ejercicio deben realizarlo en grupos de 3 o 4, pues, se trata de observar cómo son capaces los alumnos de trabajar en equipo, algo que en la práctica profesional es más que habitual.

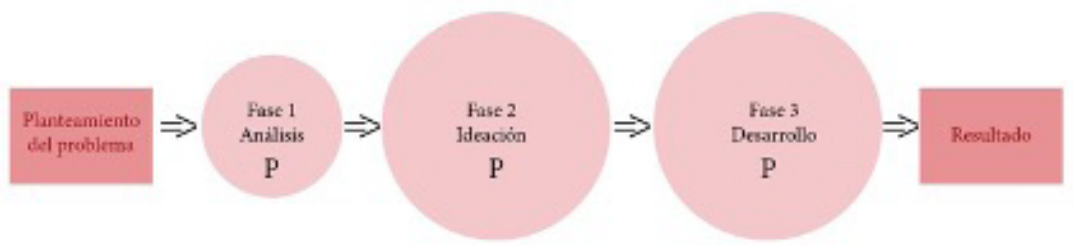

Figura 1. Modelo metodológico actual de Taller de Arquitectura. La letra P indica contenido práctico.

Por último, para una evaluación de la asignatura no se establecen pruebas escritas, sino que en cada fase los estudiantes tienen una sesión crítica final en la que cada grupo debe exponer tanto a profesores como compañeros el resultado del proyecto. La calificación y evaluación final derivan del proceso de evaluación continua a lo largo de todas las sesiones en el que se tiene en cuenta la madurez y progresión positiva del grupo relativo al proyecto arquitectónico.

Ciclos de Mejora en el Aula (2020). Experiencias de Innovación Docente de la US Esta obra se distribuye con la licencia Creative Commons 


\section{Diseño previo del Ciclo de Mejora Docente}

A diferencia de otras áreas en las que los estudiantes poseen una base de conocimientos aplicable como construcción o instalaciones, el urbanismo plantea ciertas complejidades necesarias para la intervención proyectual en las que el alumno encuentra múltiples dificultades. Debido a la experiencia en talleres previos y al análisis de las asignaturas en el grado, se ha comprobado que hay que consolidar ideas o conceptos que no han sido previamente adquiridos en asignaturas propiamente de Urbanismo. Desde Urbanismo 1 (en primero) a Urbanismo 2 (en tercero), se encuentran 3 asignaturas de talleres en las que se hacen necesarios conceptos urbanos para poder desarrollar el ejercicio presentado. Lo que implica un suplemento en el trabajo como docente para la asignatura. Por tanto, en el Taller de Arquitectura, en relación al área de urbanismo encontramos:

- Falta de conocimientos y terminologías urbanísticas concretas

- Desconocimiento de herramientas para el estudio urbano

- Necesaria aplicación de la escena urbana para el análisis y definición del proyecto.

- Escasa consideración de los estudiantes sobre la importancia del urbanismo como disciplina.

Con motivo de resolver parte de la problemática detectada en la asignatura en relación con el área de urbanística, se propone la realización de un ciclo de mejora docente en el aula de manera que se pueda otorgar a los estudiantes una visión más compleja y la adquisición de conocimientos (Bain, 2006) que en la propia asignatura rara vez tiene lugar.

Ciclos de Mejora en el Aula (2020). Experiencias de Innovación Docente de la US Esta obra se distribuye con la licencia Creative Commons 


\section{Modelo metodológico y mapa de contenidos}

Como primer punto, se proponen mejoras sobre el modelo metodológico donde el urbanismo se inserte de manera transversal y recíproca (De Alba y Porlán, 2017). En azul se incorporan mejoras en relación al modelo existente en la asignatura (Figura 2). Estas mejoras tratan de matizar que cada fase no fuese unidireccional, sino que cada una fuera un complemento de la otra. No se tratan de hitos cerrados pues todas las fases intervienen entre sí. El Análisis y la ideación es un proceso continuo que abarca todas las fases. Desde el ámbito urbano se busca implementar conceptos urbanísticos que posteriormente, en las fases 2 y 3 , sean aplicados en la escena urbana en relación a la propuesta arquitectónica. Así, las propuestas ideadas por los estudiantes estarían intermitentemente en revisión realizándose de manera cíclica el análisis, la ideación y el desarrollo desde una visión urbana.

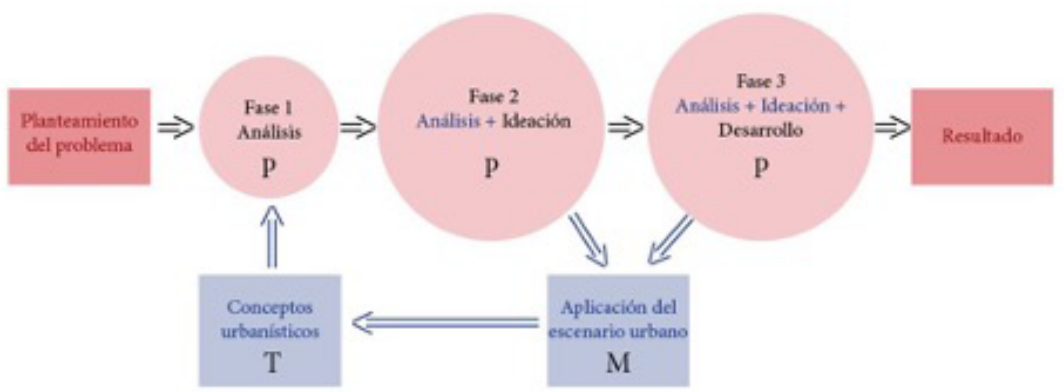

Figura 2. Modelo metodológico propuesto desde Urbanismo para Taller de Arquitectura. La letra P indica contenido práctico, la M indica metodológico y la T indica teórico.

Se procura buscar un esquema de indole circular e intentar romper con la linealidad que existe. El proceso debe ser retroalimentativo donde para partir del planteamiento del problema hasta llegar al resultado final, deben realizarse diferentes fases entre sí de manera cíclica hasta que el proyecto alcance la suficiente madurez para poder generar un buen resultado.

Ciclos de Mejora en el Aula (2020). Experiencias de Innovación Docente de la US Esta obra se distribuye con la licencia Creative Commons 
Al ser el Taller de Arquitectura una asignatura compleja en cuanto a la coordinación docente se refiere y con un enunciado de la realización de un proyecto, como cuestión desencadenante (De Alba y Porlán, 2017), por lo general la clase se plantea como correcciones a los grupos de alumnos y alumnas sobre sus proyectos. Así pues, al introducir en el modelo metodológico aspectos urbanos para intentar solventar las carencias detectadas en la asignatura con relación a esta área de conocimiento, se introducen pequeñas píldoras conceptuales con algunos contenidos que tengan lugar en el desarrollo de dos sesiones (Figura 3). Los contenidos se encuadran dentro del programa establecido en el proyecto docente, donde el epígrafe consiste en proyectar un edificio con carácter de equipamiento hospitalario en una situación de borde urbano en el barrio de Bellavista. Teniendo en cuenta tanto el modelo metodológico como los contenidos, el docente queda desplazado del centro del aula (Finkel, 2008) priorizando el trabajo de los estudiantes en grupos. Con ello, se establecen los conceptos clave que respondan a la pregunta de ¿Por qué es importante el urbanismo en el proyecto? Para ello el mapa de contenidos elaborado distingue entre Contenidos actitudinales $(A)$, conceptuales $(C)$ y procedimentales $(P)$. La pregunta que se plantea adquiere un carácter conceptual y también actitudinal ya que el urbanismo puede tener un efecto positivo o negativo en la estrategia de proyecto ( $\mathrm{Na}$ vas Carrillo, 2018). Para dar respuesta a la cuestión urbana, se introducen tres conceptos divididos en 3 componentes determinantes: emplazamiento urbano, implicaciones del entorno y movilidad urbana. Cada uno de ellos está estrechamente relacionado con aspectos tanto procedimentales como actitudinales en función de su naturaleza, llegando a compartir contenidos entre sí como el caso del Plan General de Ordenación Urbana.

Ciclos de Mejora en el Aula (2020). Experiencias de Innovación Docente de la US Esta obra se distribuye con la licencia Creative Commons 


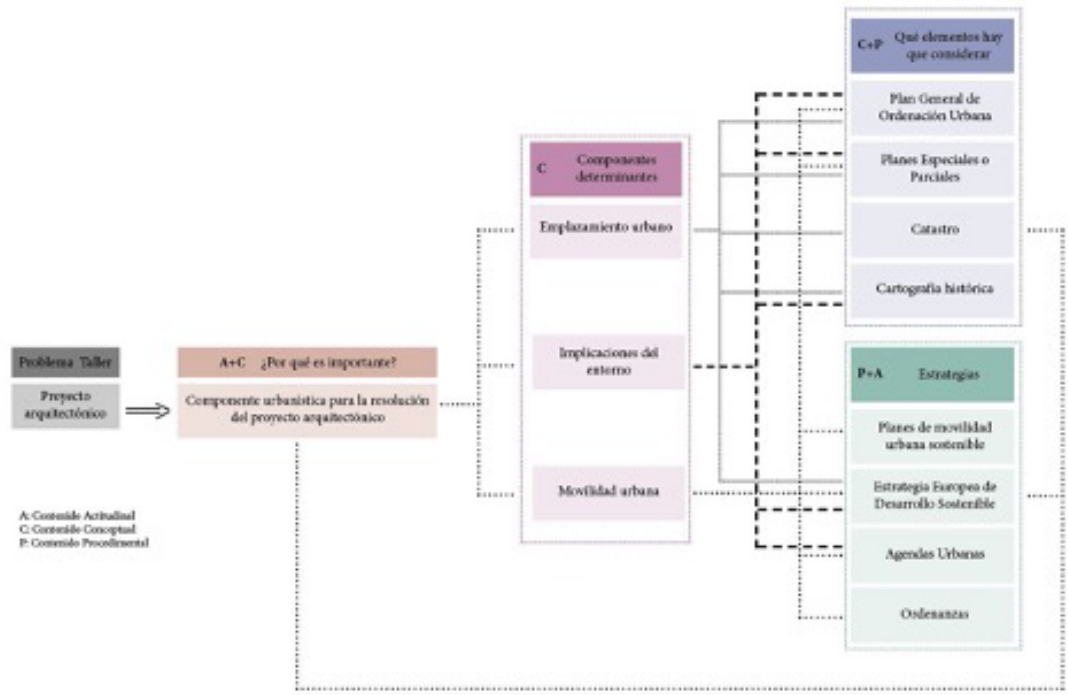

Figura 3. Mapa de contenidos establecido para el enunciado del proyecto desarrollado en el Taller de Arquitectura 3.

Hay que resaltar que los contenidos que aquí se desarrollan no competen a la propia asignatura de Taller, pero son necesarios para una adecuada resolución del proyecto arquitectónico. Para ello, el contenido se adapta en función del ejercicio planteado, respondiendo este mapa de contenido al caso exclusivo del enunciado de este Taller. Para su posterior aplicación en el ámbito del aula, se ha considerado el desarrollo de dos sesiones en las que se realicen diferentes actividades de contraste que pongan de manifiesto la naturaleza de los contenidos e impliquen el empleo de estrategias y elementos a considerar de carácter urbano para poder resolver el enunciado del Taller. Como el desarrollo de una sesión es compartida con otro profesor, se ha planteado para no interferir en la labor docente del otro profesor, el planteamiento de actividades grupales en simultáneo con el desarrollo docente del compañero que esté en clase según el cronograma de la asignatura. Al existir un calendario en la organización del curso, la aplicación de las actividades debe realizarse dentro de las horas asignadas a urbanismo (U). Por tanto se restringe

Ciclos de Mejora en el Aula (2020). Experiencias de Innovación Docente de la US Esta obra se distribuye con la licencia Creative Commons 
a las marcadas en el calendario de la primera parte. La secuencia de actividades planteadas quedaría de la siguiente manera:

Sesión 1 - (2 horas)

1.0-Cuestionario inicial y planteamiento del problema (10 $\mathrm{min})$

\section{1-Actividad por grupos (20 min)}

Señalar los límites urbanos de la ciudad de Sevilla en 4 planos: siglo XVIII / siglo XIX / siglo XX / siglo XXI. Sobre estos, definir 2 elementos que configuren bordes urbanos.

1.2-Exposición por grupos y debate (1 h 20 min) (5-6 $\mathrm{min}$ (grupo)

Cada grupo contará qué plano le ha resultado más difícil para identificar los límites y bordes, mostrando los suyos propios hacia los demás grupos.

1.3-Actividad por grupos para la próxima sesión. (Tarea para casa)

Búsqueda de un ejemplo para tomar como referencia de cómo termina la ciudad de Sevilla en 2020.

1.4-Debate sobre los resultados (10 $\mathrm{min}$ )

Sesión 2 - (2 horas)

2.1-Exposición y debate por grupos (30 min)

Sobre la actividad 1.3, cada grupo debe mostrar y exponer su ejemplo.

Ciclos de Mejora en el Aula (2020). Experiencias de Innovación Docente de la US Esta obra se distribuye con la licencia Creative Commons 


\section{2-Actividad por grupos (20 min)}

Señalar en cada una de las ciudades ejemplo, la movilidad prioritaria probable.

2.3 Debate y justificación de movilidad (50 min) 10 ciudades, $5 \mathrm{~min} /$ ciudad

Cada grupo debe identificar su elección por ciudad y entre todos, votar si es la opción más acertada.

\subsection{Debate sobre los resultados (10 $\mathrm{min})$}

\subsection{Cuestionario final (10 $\mathrm{min})$}

\section{Cuestionario inicial-final}

Para la elaboración del cuestionario inicial-final (Figura 4) se han tenido en cuenta los conceptos que se quieren trabajar, esto es, el emplazamiento, el entorno y la movilidad urbana. Al ser unas sesiones muy escasas y no haber un gran contenido, se han planteado tres preguntas que respondan a las cuestiones que aquí se plantean. Las dos primeras tienen un carácter completamente abierto en el que los estudiantes puedan expresar sus ideas iniciales y finales sobre la situación de borde urbano. Para la tercera, con el fin de poder cuantificar y medir las respuestas obtenidas, se ha procedido a realizar una enumeración de 10 elementos otorgando a su vez 10 palabras para realizar un orden. La idea es que fueran situados los diez puntos, sin que quedara ninguno vacío. Así pues, se realizan de manera anónima pero con la inclusión de la ciudad de nacimiento. Este hecho es realmente relevante en materia urbana ya que muchas de las respuestas pueden verse condicionadas por el entorno que los estudiantes han habitado. Es decir, a priori parece que las respuestas podrían diferenciarse si alguien ha vivido en una gran ciudad como 
Sevilla o en poblaciones de rango menor más vinculadas con el medio rural. Son componentes que hay que considerar por si las respuestas se vieran influenciadas por el hecho de vivir en un determinado tipo de población u otro.

Cuestionario

Ciudad de nacimiento:

1. Una ciudad podría definirse como una construcción humana en el territorio donde habita una población, compuestas por un centro, barrios, distritos,... pero ¿cómo termina realmente una ciudad?

2. Enuncia, al menos, dos elementos que consideres que configuran un borde urbano. En caso contrario, indica por qué no habría elementos.

3. La movilidad urbana compone un apartado primordial para la configuración y desarrollo de un enclave urbano. ¿Podrías colocar en un gradiente del 1 al 10 los elementos señalados, que priorizarías para una buena movilidad en la ciudad? (1 como peor y 10 como mejor).

Metro / Motocicleta / Bici / Patinete eléctrico / Bus / Coche / Tranvía / Peatón / Taxi / Tren de cercanías

1.

6.

2.

7.

3.

8.

4.

9.

5.

10.

Figura 4. Cuestionario inicial-final sobre conceptos urbanos para la asignatura de Taller de Arquitectura 3.

Ciclos de Mejora en el Aula (2020). Experiencias de Innovación Docente de la US Esta obra se distribuye con la licencia Creative Commons 


\section{Aplicación del Ciclo de Mejora Docente}

\section{Sesiones}

El desarrollo del segundo ciclo de mejora tuvo lugar en las dos sesiones planteadas y estructuradas en dos días diferentes, con una duración total de 2 horas cada una. El guion de ambas sesiones dividía la clase en una serie de ejercicios o actividades que hubieran de ser debatidas a posteri y que sirvieran de contraste con la opinión de los cuestionarios.

En la primera sesión se realizó inicialmente el cuestionario, el cual debían enviar por la plataforma al no poder realizarse en papel debido a la situación en la que se desarrolla y tener también a la mitad del alumnado de manera síncrona virtual. Posteriormente comenzó el desarrollo de la primera actividad consistente en señalar sobre planos de Sevilla los bordes urbanos de la ciudad en diferentes siglos. A cada grupo le es asignado un siglo diferente, situados entre XVIII y XXI, para posteriormente comentar y debatir de manera grupal la disposición de estos límites. Mientras los grupos desarrollan la tarea, el profesor con el que compartía la docencia realizaba su labor de corrección con dos grupos alternativamente, de manera que estos pudieran corregir y realizar la tarea al mismo tiempo. Tras la entrega de cada ejercicio, se procedió a que un grupo diferente tratara de comentar el plano de otro grupo, con el fin de ver si estaban de acuerdo no solo los componentes del mismo grupo sino entre grupos. Finalmente se pudo observar que no había ideas comunes, sino que todos estaban en desacuerdo entre sí, verificando que los límites de Sevilla no quedan del todo claros como a uno le parece cuando dibuja el plano. Sobre esta actividad llamó la atención que muchos estudiantes incluían el río como parte de la ciudad, a pesar de identificar en los 
cuestionarios a los ríos como límites urbanos. El desarrollo de la actividad duró más del tiempo establecido, debido a que el tiempo de corrección del profesor que compartía la hora se vio excedido. Por ello, el debate sobre los resultados planteados al final de la sesión no fue posible hacerlo, sin embargo si se solicitó la actividad para la próxima sesión a realizar en casa.

La actividad enviada para casa no resultó ser efectiva pues tan solo un grupo la realizó, obviando el resto el desarrollo de la misma. Quizás habría que haberla planteado directamente en clase, aunque el tiempo no era suficiente. El hecho de que la segunda sesión tuviera un carácter íntegro virtual y que los estudiantes se hayan centrado más en el desarrollo de su proyecto puede haber favorecido a que no haya tenido una adecuada implicación. En esta segunda sesión, ahora no presencial en su totalidad, se planteó la actividad sobre movilidad urbana en coordinación nuevamente con el compañero docente, siguiendo el mismo criterio que en la primera sesión. La actividad consistía en identificar el tipo de movilidad urbana, de 10 ciudades españolas que previamente habían sido analizadas por el Informe de Planes de Movilidad Sostenible en 2019. En esta sesión si se pudo comprobar las respuestas iniciales de los cuestionarios con lo que se puede aproximar una comparativa con el cuestionario inicial. El resultado fue similar al aparecido en los cuestionarios. La mayoría de estudiantes establecieron como prioritario al peatón, la bici o el autobús, siendo en algunos casos cierto, aunque impactó que el taxi tuviera una mayor impronta en 5 de las 10 ciudades planteadas. En el cuestionario inicial, prácticamente todos ellos dispusieron el taxi en los últimos lugares de la numeración establecida en el cuestionario. Por ello llamó mucho la atención de que el taxi fuera uno de los más demandados en ciudades como Madrid, Zaragoza o Málaga. No obstante, les fue explicado que ello era debido a que muchos de los desplazados a estas

Ciclos de Mejora en el Aula (2020). Experiencias de Innovación Docente de la US Esta obra se distribuye con la licencia Creative Commons 
grandes ciudades hacia las estaciones de tren o aeropuertos empleaban el taxi para la movilidad en la ciudad como vehículo más rápido. Una vez finalizada la actividad, se procedió a la realización del cuestionario final para poder realizar una comparativa con el inicial.

El tiempo restante de la actividad relativa a casa y el que derivó de la segunda sobre movilidad urbana fue empleado para correcciones siguiendo el esquema inicial planteado en el propio proyecto docente del Taller. Por ello se concluyó la sesión con las correcciones oportunas en los grupos que así lo precisaban.

\section{Evaluación}

El proceso de evaluación se ha desarrollado gracias a la realización de un cuestionario inicial y final. La idea es emplear esta herramienta presentando las mismas preguntas al principio y conclusión del ciclo de mejora para observar si realmente se ha producido un aprendizaje o evolución del pensamiento que los estudiantes poseen (Bain, 2006). Como se ha comentado previamente, este cuestionario recoge tan solo 3 preguntas pero que se encuentran enfocadas en conceptos muy concretos sobre el área de urbanismo. Para un análisis completo de la evaluación, se han tomado las respuestas dadas por los estudiantes y se han clasificado en función de ideas o categorías, sobre las que se establece una numeración y porcentaje. Estas, a su vez, se dividen en conceptos de preguntas 1 y 2 y conceptos de pregunta 3. La primera tabla (Tabla 2) divide las respuestas en cuatro niveles siendo A el nivel deseado que deben alcanzar los estudiantes y D el más alejado. Según las respuestas, las categorías se dividen en definiciones o conceptos de lo que se entiende por el borde urbano en una ciudad. Así la visión más compleja es que la ciudad no tiene bordes ni límites, mientras que la más alejada es

Ciclos de Mejora en el Aula (2020). Experiencias de Innovación Docente de la US Esta obra se distribuye con la licencia Creative Commons 
una idea de la ciudad como nos la podemos imaginar en un contexto de pasado, con murallas y acotada. En el inicio, tan solo 1 estudiante se encontraba en el nivel A quedando la mayoría sorprendentemente en un nivel B. De hecho, el nivel B es un poco contradictorio en sí porque los alumnos aseguraban que la ciudad no tenía límites pero luego definían que el río o un terreno agrícola pudiera ser un borde. Por tanto era necesario alcanzar la idea contemporánea de que el concepto de borde urbano es interpretativo ya que no existe como tal. Finalmente, muchos de los que se encontraban en el nivel B demostraron una comprensión complejizada en la que no existen bordes. Otros, por el contrario, no avanzaron a un siguiente nivel lo que probablemente fuera resultado del fruto de la confluencia de dos profesores más la modalidad semipresencial y virtual. Por último destacar que el estudiante con la idea de ciudad antigua sí que avanzó de nivel, quedando muy pocos en el C y la amplia mayoría en el A y B, lo que parece haber tenido una incidencia positiva las actividades en función a la definición de estos conceptos.

Tabla 2. Tabla de escalera de aprendizaje según datos de los cuestionarios inicial y final sobre ciudad y borde urbano.

\begin{tabular}{|c|c|c|c|c|c|}
\hline Nivel & Categoría & $\begin{array}{l}\text { Número } \\
\text { respuestas C. } \\
\text { Inicial }\end{array}$ & $\%$ Inicial & $\begin{array}{l}\text { Número } \\
\text { respuestas } \\
\text { C. Final }\end{array}$ & $\%$ Final \\
\hline A & $\begin{array}{l}\text { Ciudad sin } \\
\text { limites y sin } \\
\text { bordes }\end{array}$ & 1 & $0,5 \%$ & 8 & $42 \%$ \\
\hline B & $\begin{array}{l}\text { Ciudad sin } \\
\text { limites pero con } \\
\text { bordes }\end{array}$ & 12 & $63 \%$ & 7 & $36 \%$ \\
\hline C & $\begin{array}{l}\text { Ciudad con } \\
\text { limites }\end{array}$ & 5 & $36 \%$ & 4 & $21 \%$ \\
\hline D & $\begin{array}{c}\text { Ciudad en la } \\
\text { antigüedad }\end{array}$ & 1 & $0,5 \%$ & 0 & $0 \%$ \\
\hline
\end{tabular}

Ciclos de Mejora en el Aula (2020). Experiencias de Innovación Docente de la US Esta obra se distribuye con la licencia Creative Commons 
Además de la tabla anterior, también se trabajó el concepto de movilidad urbana sobre el cual se ha realizado una nueva tabla en el que se muestren los datos cuantificados de los cuestionarios (Tabla 3). La división en esta ocasión se realiza en 3 niveles diferenciándose por una movilidad sostenible, eficaz y no sostenible. La eficaz se sitúa en el centro ya que mezclaría aspectos tanto sostenibles como no sostenibles al tratarse de un tipo de movilidad rápida. Al inicio de los cuestionarios un 68\% situó sus respuestas en un nivel $A$, siendo muy beneficioso y absolutamente nadie en un nivel C. Por el contrario, los datos del cuestionario final resultan casi contradictorios con los iniciales quedando la mayoría en el nivel C.

Tabla 3. Tabla de escalera de aprendizaje según datos de los cuestionarios inicial y final sobre movilidad urbana.

$\begin{array}{cccccc}\text { Nivel } & \text { Categoria } & \begin{array}{c}\text { Número } \\ \text { respuestas } \\ \text { C. Inicial }\end{array} & \text { \% Inicial } & \begin{array}{c}\text { Número } \\ \text { respuestas } \\ \text { C. Final }\end{array} & \text { \% Final } \\ \text { A } & \begin{array}{c}\text { Movilidad } \\ \text { Sostenible }\end{array} & 13 & 68 \% & 6 & 32 \% \\ \text { B } \quad \text { Movilidad eficaz } & 6 & 42 \% & 5 & 26 \% \\ \text { C } & \begin{array}{c}\text { Movilidad no } \\ \text { sostenible }\end{array} & 0 & 0 \% & 8 & 42 \%\end{array}$

La respuesta no es sorprendente si se observan los datos del Informe del Plan de Movilidad Sostenible. En dicho documento se indica lo que realmente ocurre en las ciudades, no lo que debiera pasar. Esto es, el taxi y el coche aún siguen siendo los medios más empleados en el ámbito urbano a pesar de que en muchas ciudades hay una apuesta clara por el peatón o la bici. La cuestión es que se produjo un error en la comprensión de datos actuales con la búsqueda ideal del modelo de movilidad urbana en una ciudad. De ahí que se hayan obtenido los resultados

Ciclos de Mejora en el Aula (2020). Experiencias de Innovación Docente de la US Esta obra se distribuye con la licencia Creative Commons 
expresados. De nuevo, los factores del modelo de clase en pandemia y la simultaneidad de profesores no han favorecido a una correcta comprensión del contenido urbano.

\section{Evaluación del CIMA}

La aplicación de un ciclo de mejora ha requerido modificaciones en algunos puntos de la asignatura como cambios en la metodología, la creación de un tipo de contenido e incorporación de actividades en dos sesiones. Todo ello considerando el actual programa docente de la asignatura y procurando no establecer conflictos con el desarrollo docente de los profesores que se encuentren en simultáneo durante su desarrollo.

Para la aplicación del ciclo de mejora modelo metodológico inicial hubo de incorporar cambios respecto al original para intentar introducir conceptos urbanísticos necesarios para el desarrollo del ejercicio propuesto. En este sentido, inicialmente se planteaba un problema, se iniciaba una fase 1 de análisis, posteriormente, una fase 2 de ideación y una fase 3 de desarrollo. Por ello, para que en las fases 2 y 3 apliquen los estudiantes de forma correcta una implantación adecuada del escenario urbano, era necesario introducir conceptos urbanísticos en la fase 1. También se ha incluido el análisis como parte de todas las fases y no solo de la primera, para fomentar que se realice una constante revisión ya que no se trata de un proceso estanco. A su vez, la idea de proyecto también es constantemente revisada por lo que en la fase 3 sería un compendio de la 1 y la 2 sumándose el desarrollo, para así obtener el resultado final que es la ejecución del proyecto de arquitectura propuesto.

El cuestionario inicial-final ha resultado ser una herramienta con bastante utilidad. Los estudiantes se han expresado con total sinceridad reflejando sus ideas sin

Ciclos de Mejora en el Aula (2020). Experiencias de Innovación Docente de la US Esta obra se distribuye con la licencia Creative Commons 
temor alguno en los cuestionarios. Algo que hubiera sido de una forma totalmente opuesta si se les hubiera preguntado en la propia sesión directamente, ya que se enfrentarían a la presión tanto del contexto del aula como del docente. A su vez, el debate en grupo y entre grupos resultó también muy efectivo de manera que ellos mismos pudieran opinar sobre sus compañeros, lo que los sumergía más en el propio debate.

Se ha encontrado cierta dificultad en la entrega del cuestionario, de un total de 25 estudiantes, solo 19 lo han enviado. Desconozco si el motivo se debía a encontrarse la mitad en clase y la otra mitad de manera virtual debido al modelo semipresencial, aunque todos ellos tenían conocimiento de su realización. Quizás hubiera sido por anunciar que no era una actividad calificable y eso hizo que muchos de ellos obviaran su envío directamente. La gestión del tiempo ha sido difícil en ambas sesiones, bien porque algunas actividades han durado poco o porque se hayan extendido. A su vez, la actividad en casa tampoco resultó efectiva ya que el problema y/o enunciado de la asignatura es la realización de un proyecto arquitectónico. Al tener un enunciado muy específico, que a su vez requiere de bastante tiempo, considero que los alumnos pensaron que quizás una actividad "extra", sin calificación, no era necesario realizarla. En este sentido creo que sería conveniente haberla aplicado directamente en la sesión de clase, aunque por falta de tiempo, quizás se hubiera suprimido. Respecto al tema de la movilidad urbana, me percaté tras realizar el cuestionario final que resultado obtenido hubiera podido ser por el perfil de usuario urbano que representan como "estudiantes", por lo que priman medios como la bici o el autobús. Si quizás el perfil encuestado hubiera sido diferente, como ocurre en el informe de movilidad, la respuesta hubiera diferido. Posteriormente, tuvo su reflejo en el cuestionario, donde la gran mayoría colocaron el taxi en una posición superior. Ello conllevó a una malinterpretación creyendo que era mejor para la ciudad según los datos basados en el informe de 2019. Finalmente, en la 
siguiente sesión procuré corregir la idea errónea que pudiera surgir sobre este medio de transporte incidiendo en la importancia de una movilidad sostenible.

Sobre el ciclo de mejora se han realizado un par de conclusiones en relación con el ejercicio general del Taller de Arquitectura. En la asignatura se realiza desde el primer día la ejecución de un proyecto de arquitectura, dentro del cual se inserta desde el área de urbanismo la oportunidad de incorporar conceptos urbanísticos y que estos fueran de aplicación en el propio proyecto del taller. Aunque en las sesiones se generaron buenos resultados e incluso se produjo una evolución más compleja del pensamiento de los estudiantes entre los cuestionarios inicial y final, el resultado no ha sido el esperado. Los estudiantes han comprendido mejor los conceptos desarrollados pero no son capaces de incorporarlos en el proyecto de arquitectura. En este sentido, creo que habría que mejorar en la aplicabilidad directamente sobre el proyecto. Este hecho se ha podido comprobar debido a que tras finalizar el segundo ciclo, los estudiantes continúan las mismas estrategias de proyectos sin un carácter urbano como el comentado durante el ciclo. Por ello, se procurará no tanto en la comprensión del concepto sino en su aplicabilidad. Aunque se hace difícil ya que no se dispone de mucho tiempo por lo que habría que reordenar las tareas y reestructurar el desarrollo de las sesiones.

\section{Conclusiones}

El Taller de Arquitectura supone un reto en sí para la aplicación de un ciclo de mejora debido a las dificultades y oportunidades que la propia asignatura plantea desde su inicio. La ausencia de un contenido específico permite que en muchos casos se produzca una ausencia de investigación, tendiendo los estudiantes que enfrentarse de manera autónoma a un tipo de enunciado muy concreto. A veces se repiten modelos analíticos, sin saber realmente

Ciclos de Mejora en el Aula (2020). Experiencias de Innovación Docente de la US Esta obra se distribuye con la licencia Creative Commons 
por qué, derivando en una búsqueda de información banal sin relación con el proyecto. Así, a través de una interacción más cercana, preguntas clave y actividades de contraste adecuadas, se puede complejizar y hacer evolucionar el modelo mental de los estudiantes sobre el área de urbanismo.

Durante la enseñanza del Taller de Arquitectura los estudiantes deben poner en práctica lo aprendido en otras asignaturas de manera conjunta y unitaria para la realización de un proyecto de arquitectura. Según el problema que se plantea cada curso, es decir, el tipo de proyecto arquitectónico, el contenido que se propone de enseñanza se modifica. En lugar de basar el aprendizaje en enseñar contenidos, se procura que los estudiantes comprendan conceptos "clave" que sean empleados como soporte de la idea de proyecto. Para ello se han elaborado actividades, principalmente a través de planos y/o proyectos de referencia, para que los estudiantes analicen, comparen y trasladen a su propia problemática. Además se trabajan ideas que han sido desarrolladas en otros proyectos de igual índole y que pueden ser aplicadas en el propio proyecto del estudiante. El objetivo es que ellos entiendan el concepto que se quiere poner en práctica, lo interpreten y lo ejecuten como estrategia en el proyecto de intervención.

Habría que destacar la importancia de la aplicación del ciclo de mejora no solo como una experiencia sino como una oportunidad para poder mejorar la práctica docente. Entre otras claves que ha podido aportar al desarrollo de la asignatura ha sido los cambios aparecidos en la metodología, la posibilidad de incluir contenido en la asignatura y sobre todo el empleo de los cuestionarios como herramienta efectiva para poder tener una idea clara de las verdaderas idas de los estudiantes. No obstante, estos cambios se han realizado principalmente en las primeras fases de análisis e ideación, lo que la evaluación 
total de la asignatura debe responder a un proceso global y pleno de las actividades desarrolladas. La evaluación y por ende, la calificación, deben realizarse sobre un ejercicio final presentado, donde los aspectos urbanos representan 1/ 6 de la evaluación total, al existir cinco áreas más de conocimiento. Ello establece una dificultad aún mayor en la asignatura, que en el caso de poder plantearse un ciclo de mejora completo e incorporado en la práctica docente debiera de incluir a 6 profesores, lo que sin duda sería aún un mayor reto pero supondría quizás un mayor enriquecimiento de la docencia universitaria en el Taller de Arquitectura.

A partir de la enseñanza del Taller de Arquitectura y el desarrollo del Ciclo de Mejora se elabora un listado de principios didácticos personales basados en la propia experiencia docente. Como finalidad, el problema que se ejecuta conecta directamente con la realidad al tratarse de una simulación real del desarrollo de un proyecto en un escenario concreto. En relación a los contenidos, estos no tienen una elevada relevancia por lo que se ciñen a conceptos "clave" y valores actitudinales en relación a la manera de abordar la propuesta. Como metodología se ha basado en la resolución del proyecto de arquitectura a través de un proceso cíclico de análisis e investigación. La evaluación se propone como un seguimiento del proceso de los estudiantes, aunque esta se ciñe no solo a uno sino a seis profesores, lo que se traduce en buena manera en una concentración de la calidad y desarrollo del ejercicio final. Las relaciones profesor-estudiante procuro que sean lo más próximas posibles intentando que se cuestionen conceptos e incentivando el debate. Sobre el aprendizaje se procura intentar decir aquello que no se ha comprendido de un modo diferente, repitiendo la idea desde otro ángulo o perspectiva. No obstante, se anima a que los propios estudiantes investiguen por si solos y en el caso de duda seguir la orientación del profesor. Sobre la teoría del

Ciclos de Mejora en el Aula (2020). Experiencias de Innovación Docente de la US Esta obra se distribuye con la licencia Creative Commons 
conocimiento, siempre pongo en duda todo aquello estipulado como principio inalienable haciendo reflexionar sobre el mismo y que este no es inquebrantable, sino que debe ser argumentado en función a un criterio propio.

Por último se incluye una reflexión personal sobre el proceso de realización del ciclo de mejora ya que este supone un avance en el proceso de desarrollo personal como docente. Es importante matizar la importancia de una continua formación en el aprendizaje del rol como docente, conocer cómo hay que enfocar una clase independientemente del área de estudio. Las tareas docentes abarcan mucho más allá que la propia clase, ya que requiere tiempo, esfuerzo, compromiso y vocación, elementos que empujan al docente a realizar un ciclo de mejora que poco a poco ha de ir matizando y mejorando. Se tratan pues, de habilidades que la experiencia va otorgando en la docencia universitaria, las cuales son claves para el futuro no solo del profesorado sino de los propios estudiantes.

Ciclos de Mejora en el Aula (2020). Experiencias de Innovación Docente de la US Esta obra se distribuye con la licencia Creative Commons 
Palabras clave: Taller de arquitectura 3, Grado en Fundamentos de la Arquitectura, docencia universitaria, experimentación docente universitaria, urbanismo.

Architecture Workshop 3, Degree in the basis of the architecture, university education, university teaching experimentation, urban planning.

\section{Referencias bibliográficas}

Bain, K. (2006). Lo que hacen los mejores profesores de universidad. Valencia: Servicio de Publicaciones de la Universitat de València.

De Alba, N. y Porlán, R. (2017). La metodología de enseñanza. En R. Porlán (Coord.) Enseñanza Universitaria. Cómo Mejorarla (pp. 37-54). Madrid: Editorial Morata.

Finkel, D. (2008). Dar clase con la boca cerrada. Valencia: Servicio de Publicaciones de la Universitat de València.

Navas Carrillo, D. (2018). Ciclo de mejora docente en el Área de Urbanismo y Ordenación del Territorio. Medidas de protección frente a la contaminación visual y perceptiva en el patrimonio urbano. En R. Porlán y E. Navarro Medina (Coord.), Jornadas de formación e innovación docente del profesorado (pp. 482-499). Sevilla: Editorial de la Universidad de Sevilla.

Universidad de Sevilla (2020a). Proyecto docente Taller de Arquitectura 3. Departamento de Proyectos Arquitectónicos. Escuela Técnica Superior de Arquitectura. Curso 2020-2021.

Universidad de Sevilla (2020b). Plan de actuación específico en el escenario " $A$ " de la ETS Arquitectura de la Universidad de Sevilla. Escuela Técnica Superior de Arquitectura. https://etsa.us.es/sites/arquitectura/files/Escuela/Recursos\%20y\%20Servicios/COVID19/ PLAN\%20ESPECIFICO\%20ACTUACION\%20ARQUITECTURA\%20v8\%2020200928.pdf (20/11/2020)

Ciclos de Mejora en el Aula (2020). Experiencias de Innovación Docente de la US Esta obra se distribuye con la licencia Creative Commons 